\title{
CEREBELLO-BULBAR POLIOENCEPHALITIS ORIGINAT- ING DURING OR AFTER EPIDEMICS OF INFLUENZA AND OF - POLIOMYELITIS
}

INCLUDING THE RECORD OF A CASE OF EPIDEMIC ENCEPHALITIS OF THE LETHARGIC TYPE *

CHARLES K. MILLS, M.D., LL.D.

Neurologist to the Philadelphia General Hospital and Emeritus Professor of Neurology in the University of Pennsylvania

AND

GEORGE WILSON, M.D.

Assistant Neurologist to the Philadelphia General Hospital and Instructor in Neurology in the University of Pennsylvania

PHILADELPHIA

Three of the six cases recorded in this paper occurred during or after the recent prevalence of influenza, the history of an influenzal attack appearing to precede the occurrence of the involvement of the nervous system. The other three cases were observed at times when epidemic poliomyelitis prevailed. Five of the cases were clearly instances of focal encephalitis. The fifth case was doubtfully one of cerebello-bulbar encephalitis. In several of these cases the differential diagnosis most considered was that of cerebello-pontile tumor and localized encephalitis. A close scrutiny of the general symptoms of the cases, however, indicates that these were rather of an infection or toxi-infection than of a progressively increasing neoplasm. The latter was presumed in several of the cases, for a time at least, because it was held that the symptoms gradually augmented. Strickly speaking, this was not true, although the symptom picture was not completed as quickly as is usual in poliomyelitis or polioencephalitis. The sixth case was demonstrated pathologically to be one of epidemic encephalitis and progressed from the onset until death in about ten days. In the other five cases the course of the affection in its acute stage, however, corresponded to what we have not infrequently seen, namely, a rapid development of a part of the syndrome with a subsequent history of extension and recrudescence.

* Read before the meeting of the Philadelphia Neurological Society, March 28, 1919. 
The study of these cases suggests, among other things, the relationship of epidemic poliomyelitis and epidemic influenza, especially as regards the involvement of the central nervous system in the latter disease. The literature which discusses the symptomatology and pathology of polioencephalomyelitis is very extensive, and will not be considered in this connection, except to refer briefly to the special types of poliomyelitis or focal encephalitis illustrated by the cases described.

Batten $^{1}$ has contributed an elaborate paper, brief references to which will be sufficient to cover most of the points we desire to bring out. A full bibliography of the subject is appended to his paper. Discussing the bulbar and pontile forms of the disease, Batten refers to Bremer's report that 12 per cent. of 400 cases showed evidences of encephalitis, involving the oblongata, pons or mid-brain. Facial paralysis was the commonest manifestation, while cases with ataxia, nystagmus and tremor formed the next most numerous group. Besides the facial nerve nuclei, those supplying the tongue, the palate, the masseter and temporal muscles were sometimes affected, either unilaterally or bilaterally. Lesions of the oculo-motor nuclei occurred, giving rise to a complete or partial ophthalmoplegia. Blindness was sometimes associated with the ocular paralysis. The observations of Batten and the others whom he cites on the blindness sometimes seen in poliomyelitis, and the rhythmic tremor associated with ocular and other cranial nerve palsies which occur in mid-brain lesions, are of much interest, but are not within the scope of the present contribution.

Cases are referred to by Batten in which ocular palsies with nystagmus were present and still others in which there was evidence of involvement of the fifth, sixth and seventh cranial nerves, the variation in symptoms depending on the situation and extent of the lesion.

Describing the cerebellar or ataxic form of poliomyelitis, Batten states that this type is characterized by the acute onset of ataxia, sometimes associated with ocular and other cranial nerve paralyses and alteration of articulation. Nystagmus was often absent. In some cases the cerebellar ataxia cleared up quite rapidly, and in others it took many months or years.

Batten and Wickman recognize the "neuritic" form of poliomyelitis to which we make reference because in one of the cases of the following series both cerebellar and neuritic symptoms were present.

1. Batten, F. E.: Acute Poliomyelitis, Brain, Lond., Parts 1 and 2, 39: (June) 1916. 
Batten refers to the occurrence of both second attacks of poliomyelitis and of relapsing cases. Our experience is that relapsing, or what is a better term, recrudescent forms of the disease not infrequently occur, as illustrated by cases in the following series. The patient after a first attack, which may be of moderate severity, has a decided increase or renewal of fever which results in marked cerebellar or cerebellar and paralytic phenomena. Besides these recrudescent or relapsing forms of cerebellar and other types of poliomyelitis, cases occur which from the first take on a course different from that usually described in the typical instances of the disease. They assume a sub-acute or sub-chronic character, the probable nature of the lesions not being fully exhibited for some weeks.

CASE 1.-Child 3 years old; influenzal attack followed by weakness and awkwardness in the use of the lower extremities; recrudescence of acute symptoms after three months; marked cerebeilar asynergy affecting both lower and upper limbs; alternating strabismus and nystagmoid movements; no papilloedema; gradual improvement.

History. - This patient was a girl aged 3 years, who was brought from Vickburg, Miss., in the latter part of February of the present year. Last October she had an attack believed to be influenza, with high temperature, the acute symptoms lasting one to two weeks. The child had a tendency to be unsteady on her feet after she rerovered from the febrile attack, getting gradually worse. About January 20 , the patient began to have severe vomiting, after she had overloaded her stomach with apples and other food. She continued to be worried by her stomach for about ten days and about twelve days after the spell of vomiting, began to have great difficulty in standing and walking, stumbling and falling. In a week or less she was unable to stand or even to sit up straight. During the week before she was first seen by us the mother thought she had improved somewhat in her power of sitting up.

Examination.-This showed inability to stand alone, unless it was for a scarcely perceptible fraction of a minute. When she walked with assistance she kept the legs rather wide apart and was unable to guage her position or stand with firmness. She had a little more control over the left than the right side. When she attempted to feed herself with a spoon she had diffculty in doing so, because she was awkward and did not reach her mouth accurately.

Symptoms.-Briefly stated, the patient's most important symptoms were a cerebellar station and gait, with also some marked cerebellar asynergy in the upper extremities-more pronounced in the right than in the left. She had also some involvement of her ocular movements of the nature of an alternating strabismus with nystagmus or pseudo-nystagmus, although this seemed to be rapidly disappearing. Dr. J. W. Croskey, the ophthalmologist who examined her, reported that she had no papilloedema. 
Clinical Course and Outcome.-This patient remained under observation about ten days or two weeks, improving slowly but steadily in the use of her limbs and trunk.

The examination showed none of the general symptoms of brain tumor. The history indicated a febrile attack of some severity, leaving the child weak and awkward in her movements and a subsequent seizure with vomiting, after which the complete inability to stand and walk resulted. If the sickness in October was influenza with some poliomyelitis of the focal encephalitic type, a relapse or recrudescence of the febrile affection probably occurred three months later.

CASE 2.-Man about 30 years of age; infuenza with temperature from 100 to $102 \mathrm{~F}$; headache, vomiting, vertigo and diplopia; cerebellar station and gait; paralysis of right external rectus and of right facial nerve; nystagmus on horizontal excursion of eyes to right or left; no papilloedema; rapid improvement with recovery in about one month.

History.-This patient was a physician, aged about 30, who recently had been living and practicing in West Virginia.

The patient stated that he had stopped work two weeks before he came under observation. For several days before that time he had not been feeling well. His temperature had been running from 100 to $101 \mathrm{~F}$. $\mathrm{He}$ went to bed with a temperature of about 102, headache, and hyperesthesia over the left chest and right side of the face. He also suffered from dizziness and vomiting in the morning. He had some diplopia on looking to the left. His headache continued.

Examination.-Dr. W. T. Zentmayer reported that the patient had paralysis of the right external rectus and partial paralysis of the right side of the face. The fields of vision were normal. The patient was myopic, but had no papilloedema. Some nystagmus was developed in looking either to the right or left.

Our examinations confirmed the paralysis of the right external rectus and paresis of the right facial nerve. No motor fifth paralysis and no impairment of hearing were present. Nystagmus was developed in looking either to the right or left. He had a Romberg symptom with eyes closed and showed decided unsteadiness in walking. No hypermetry, or adiadokokinesis, or tremor was present. Knee jerks were very prompt, but a Babinski response was absent. He had no clearly determined objective loss of sensation, although at times he thought he felt a little better on the left than on the right side of the face.

Clinical Course and Outcome.-This patient went to Atlantic City where he remained for two weeks. In a day or two the paralysis of the right external rectus began to improve and in a few days had entirely disappeared. At the same time that the abducens paralysis improved, the loss of power in the seventh nerve distribution began to grow worse and in a few days was so marked that the patient exhibited almost a complete paralysis of the upper and lower branches of the facial nerve. In about a week this paralysis also began to clear up and when he was seen at the end of two weeks from the time when he was first examined, both the sixth and seventh nerves were practically normal. He still had a few nystagmoid movements in looking either to the extreme right or to the extreme left. Testing him for station and gait he was now practically normal, although he thought he had a little tendency to trend to the right in walking. 
The history and examination of this patient indicated an influenzal attack with focal encephalitis, involving the cerebellum and bulb. Evidently, however, little destruction of nervous tissue took place, for the patient improved and in less than a month was almost normal.

CASE 3.-Severe febrile attack, cither influenzal or poliomyelitic, in a girl of 11 years; headache; uncertainty in station and gait, probably cerebellar; pain in limbs, trunk and head; some zeakness in abduction and dorsal flexion of feet with partial steppage gait; probable involvement in a poliomyelitic process of cerebellum and peripheral nerves; recovery in a few weeks.

History.-This patient was first seen by Dr. Mills, Jan. 17, 1912. She was then 11 years old. Four months previously she had a sharp attack of fever, supposed at the time to be typhoid, although this was doubtful. She had headache, chill and fever and at the end of about a week, when she was first seen by the family physician, her temperature was found to be $104 \mathrm{~F}$. The high temperature lasted for two days and two nights. The attack was probably one of influenza or epidemic poliomyelitis. She was kept in bed for about a week. When she got on her feet she was found to be weak and uncertain in her station and gait. In a day or two she was able to walk fairly well, but from time to time had a return of the headache. About three weeks before she came under observation she became decidedly weak in the legs and had severe pain in the feet and legs, and occasionally shoots of pain in her trunk, arms and head.

Examination. - When first examined she had an uncertain station and peculiar gait. In walking she straddled, keeping the feet apart. She guided her steps by keeping her eyes on the floor. Examination for all the muscular groups of the lower extremities showed all movements preserved, but some weakness in abduction and dorsal flexion of the feet, these giving her what seemed to be a partial steppage gait. Her knee jerks were decidedly plus. She had a slight foot clonus on the right and no Babinski response on either side. Sensation was unimpaired and she had no nystagmus. She had no disorder of speech. Pupils were normal and she had no ocular, facial or upper limb palsies and no ataxia in the finger-to-nose test or the heel-to-knee test. The Wassermann test gave a very doubtful positive reaction.

The following opinion was given regarding this case:

"The history of the febrile attack looks more like a poliomyelitic (or influenzal) seizure than one of typhoid fever. The parents said the fever lasted only two or three days and the child was only in bed a week or a little more.

"The symptoms are peculiar and unusual. The child is somewhat ataxic and seems to have some weakness, but no paralysis, in the movements of abduction and dorsal flexion of the feet. The case may have been one of the extremely unusual instances of poliomyelitic cerebellar disease, that is, some inflammation and destruction of the cerebellum and other parts may have taken place. The pains in the limbs look toward a neuritis. On the whole, the prognosis is not altogether bad as the child may have reached the limit of her serious symptoms."

Clinical Course and Outcome.-This patient was seen for a few times for several months. She then passed entirely out of observation until February of this year, when she was brought for headache, mental depression and 
irritability of the bladder. Examination for the old symptoms showed that they had entirely disappeared. Station and gait were normal. The Wassermann report was negative.

Final consideration of this case indicates that it was probably one of poliomyelitis, conjointly of the cerebellar and polyneuritic type, similar to cases referred to by Wickman and Batten.

CASE 4.-A woman about 30 years of age; numbness and some loss of power in the right half of the body developed suddenly, slowly increasing; six weeks after onset symptoms began to shoze marked exacerbation; examination showed on the left side paralysis of the seventh and sixth nerves, paresis in the motor fifth, and loss of hearing; vertical nystagmus and complete loss of lateral movements of the eyes; some loss of corneal sensibility on the left; sense of position and stereognostic perception lost; touch, pain, temperature and localization senses impaired on the right side; some ataxia in the finger-to-nose and heel-to-knee tests on the right; paresis in limbs of right side, with exaggerated knee jerk, foot clonus and Babinski reflex; perspiration localized in the right side of face and neck; gradual improvement in all symptoms, except the facial paralysis, the upper distribution of the seventh nerve being more markedly paralyzed than the lower.

History. - This patient was a single woman, aged about 30 , who was admitted to the University Hospital on September 7, where she remained until Nov. 12, 1911. The case was one of much severity and of great interest as regards the question of diagnosis. Cerebello-bulbar tumor, meningitis and occlusion were suggested.

Until about July 4, a little more than two months before coming under observation, the patient had been quite well. One day she somewhat suddenly became faint and experienced numbness in the right side of the face and in the right arm and leg. She noticed also at the time some loss of power in the arm and leg affected. Gradually the power of the arm and leg became less. Until two weeks before coming for treatment, that is, about six weeks after the onset of her symptoms, she was able to get around without aid, although her vision was somewhat impaired. At this time, while on a shopping trip, her vision failed quite noticeably during the day. Her face was drawn up somewhat on the right side and her speech was affected. During the next ten days she grew gradually worse and three days before her admission into the hospital her symptoms rapidly increased.

Examination.-Neurological examination revealed the following: Complete left-sided peripheral facial paralysis, tongue going to the right on protrusion, and slight difficulty in swallowing water. Opening the mouth the jaw went to the left, showing motor fifth paresis. Movements of the masseters and temporals on the left were weak.

Sensation was normal in the fifth distribution on both sides, except for some loss of corneal sensibility on the left. The sense of position was lost in the right arm and leg, and touch, pain and temperature showed impairment in these limbs, this being somewhat more marked for touch. Marked astereognosis was present in the right hand. The sense of location tested for the right hand was much impaired. Active and passive movements were markedly diminished in the limbs on the right side, but not lost. Right grip was poor. Marked foot clonus was present on the right and the Babinski response was elicited with difficulty on the same side. The right side of the face and neck perspired profusely, the left remaining dry. Slight ataxia was shown in the finger-to-nose and heel-to-knee tests. 
On several occasions from the time of this patient's admission to the University Hospital and as late as four years after this time the eyes were carefully examined and reported on by Dr. E. A. Shumway. The first report by Dr. Shumway made on the day of the patient's admission to the hospital, Sept. 7, 1911, was as follows:

"Spasmodic closing of right eyelids; left eyelids paretic, unable to close them. Pupils contracted; left, pin point size; right, about $1 \frac{1}{2} \mathrm{~mm}$. React to light. Vertical nystagmus, greatly increased in looking upward or downward. These movements are well carried out ; complete absence of all lateral movements of eyes. Eyegrounds entirely normal; no blurring of nerve edges or disturbance of retinal circulation. Right vision, about $4 / 25$; left vision, the same."

A week later Dr. Shumway reported: "Pupils still contracted; vision 4/20; apparently myopia from ciliary spasm. Eye-grounds show no changes. Refraction is low hyperopic astigmatism."

Other reports made by Dr. Shumway during the month after the patient was admitted to the hospital were as follows:

Sept. 25, 1911: "Returning movement of eyes toward right, especially of the right eye, which moves outward and downward. Left eye moves to the right, but much less well than right eye."

Sept. 26, 1911: "Right eye shows blurring of upper and lower edges of disk margin; no venous tortuosity. Left nerve slightly blurred similarly."

Oct. 6, 1911: "Nerves show no further blurring. Pupils less contracted. Movement of right eye toward right improved. No movement of eyes to left."

Examinations of her ears, nose and throat were made by Dr. Ralph Butler, who reported as follows:

"Pharynx examined for sensation showed practically no difference on either side of throat. In the larynx there is no paralysis either unilateral or bilateral, of vocal cords. Left drum head moderately retracted, especially the posterior half ; mobility excessive, especially the posterior half. Tenderness along insertion of sterno-cleido-mastoid. Hearing for watch tick, air and bone conduction lost on the left; normal on the right. With tuning fork the patient recognized vibration on left, hears on right. Bárány's thermic nystagmus test-no result produced in the left ear (temperature $78 \mathrm{~F}$.) ; maybe due to loss of lateral movement. Normally under this test both eyes should move to the right."

Clinical Course and Outcome.-It would be tedious and somewhat unprofitable to trace the history of this patient day by day or week by week from the time she was admitted to the University Hospital. An effort will therefore be made to summarize the results of observation and treatment. After the first few days gradual improvement began, this especially showing itself in returning power in the right extremities with concomitant improvement in the ataxia and sensory impairments. In a little less than two months the patient was able to stand and move around with difficulty. Her vision and loss of associated movements improved steadily. The facial paralysis persisted, as did also that of the sixth nerve. The seventh nerve paralysis was unusual in the fact that the upper distribution of the nerve was more pronouncedly affected than the lower, as shown by the loss of power in the frontalis and orbicularis palpebrarum. The lower distribution was also 
paralyzed, but the patient in a comparatively short time had a return of the power to draw the face to the left and retract and elevate the corner of the mouth. The patient has continued under observation for years, being seen at comparatively long intervals-the last time, about a year ago: Eventually she recovered almost entirely, with the exception of the seventh nerve paralysis.

This case, in some respects one of the most interesting of the series, viewed in the light of observations extending over years, was clearly one of poliomyelitic destruction, cerebellar, bulbar and pontile. Like the case which follows, it for a time strongly suggested the diagnosis of tumor. Operation was at one time discussed and dismissed.

Several diagnoses were suggested in this case, these being tumor, meningitis, occlusion of the posterior inferior cerebellar artery, and encephalitis affecting the cerebellum, oblongata and pons. Tumor and meningitis were dismissed after consideration, the general symptoms of these diseases being absent. There was much in favor of the diagnosis of occlusion, especially corneal anesthesia on the left side and impairment of sensation, cutaneous and deep, in the opposite half of the body. The persistent destructive symptoms, however, were those which involved the motor cranial nerve nuclei and tracts and the left pyramidal system was evidently to some extent implicated. Sensory symptoms have been recorded in association with the motor nuclear and pyramidal signs in epidemic encephalitis, and even in spinal poliomyelitis. The course of the disease seemed to us rather to indicate an inflammatory process, at first of subchronic or subacute character, with later severe exacerbation. The sensory symptoms cleared up in a comparatively short time, while those referable to the motor cranial nerves, especially the seventh and sixth, persisted. the facial paralysis remaining permanently. On the whole, it seems to us that the diagnosis of a cerebello-bulbar encephalitis of the poliomyelitic type was most probable.

CASE 5.-A boy, five and a half years old; an attack of nausea and vomiting lasting several days; diplopia, weeakness of the right side of the face, and azvkward station and gait; paralysis of the right external rectus; no papilloedema; examination shorved involvement of the fifth, sixth, seventh and eighth nerves; hypermetry, adiadokokinesis and tremor on the right side; deep reflexes exaggerated on the right; on the left, foot clonus and Babinski response; chief symptoms cerebello-bulbar on the right with some pyramidal symptoms on the left; no sensory manifestations; Bárány cxaminations were reported as indicating probable brain tumor; a first operation seemed to disclose a cerebello-pontile tumor; second operation made this doubtful; patient died, but no report on necropsy was obtained. 
History.-The patient, a child, $5 \frac{1}{2} 2$ years old, was reported to have been very healthy until the age of 2 years when he had an attack of malaria, after which his appetite and general health were somewhat impaired. Six weeks before coming under observation he had an attack of nausea and vomiting which lasted several days. He may have had fever, but no record of this was received by us. After this attack he saw double. Some weakness of the right side of the face was noticeable and he stood and walked awkwardly. The patient was referred to Dr. Frazier and Dr. Mills by Dr. B. R. Tucker with the idea of having an operation should the case be decided to be one of neoplasm. Ophthalmic examination showed paralysis of the right external rectus and no papilloedema.

Examination.-The following is a summary of some of the results of the examinations of this patient:

In the first place, he had cranial nerve involvement-fifth, sixth, seventh, and eighth. Cerebellar symptoms were on the right side, hypermetry, adiadokokinesis and tremor. His deep reflexes were exaggerated. The knee jerks and Achilles jerks were present and active. On the opposite side, he had a true Babinski response and an abortive foot clonus. The examination, in brief, showed some probable involvement of the pyramidal tracts on the right, although the chief symptoms were cerebello-bulbar and pontile on this side. Sensory changes were entirely absent. The examination of the eyes of this patient by Dr. S. D. Risley showed no papilloedema, no pupillary abnormalities, and no ocular symptoms, except the paralysis of the external rectus. Bárány examinations were made by Dr. Isaac $\mathrm{H}$. Jones, who reported his belief that these indicated a cerebello-pontile growth.

Clinical Course and Outcome.-With regard to the results of the examinations, these did not seem to be conclusive for brain tumor. Dr. Jones believed that there was a tumor of about an inch in length, reaching as high as the cerebral crura, but it seemed to us that a destructive lesion involving the nuclei and tracts of the nerves indicated by the symptoms might as readily explain the case. It was noticeable in this case also that the symptoms, with the exception of the involvement of hearing, were all motor and it seemed hardly probable that a cerebello-pontile tumor of the size apparently indicated would not cause some impairment of sensation, directly or by pressure. Such a tumor would almost certainly cause papilloedema and yet both fundi remained normal.

It was finally decided to operate. Dr. G. P. Müller performed two operations on the case. The first was a suboccipital craniectomy on the right side. The surgeon reported that there was probably a tumor of the right cerebellopontile angle, over which certain of the nerves were stretched. It was considered at the time of this operation inadvisable to go further. About two weeks later a second operation was performed. The nerves were located, but now no tumor could be seen or felt. At the site of the pons and in the angle the brain had a grayish appearance as though an inflammatory reaction had occurred at this point. The surgeon felt that the boy had either some form of meningitis which had healed and left him with an exudate, or else that he had a tumor deep in the brain causing the symptoms.

This patient died a short time after returning to his home in Virginia, but we were not able to obtain any record of a necropsy. We regard the case as of much doubt from the standpoint of diagnosis. While there was much in favor of the diagnosis of a cerebello-pontile tumor, contradictory data were equally marked. 
CASE 6.-Patient, female, 17 years old; illness began with diplopia, headache and vomiting; patient developed a stuporous or semi-conscious state from which she could be aroused by prodding; upper eyelids never elevated; catatonic state; some rigidity of the neck; horizonal nystagmus; left external rectus paretic; no papilloedema; all muscular movements slow and studied; deep reflexes absent or very zeak; no Babinski reflexes; temperature ranged from 99.3 to $102.4 \mathrm{~F}$.; urine and blood were normal; patient died on about the tenth day of illness; after hardening, naked eye examination showed softening and small hemorrhages in cross sections of the midbrain, around the aqueduct of Sylvius, and in the upper part of the pons; sections from the midbrain and the pons showed many minute hemorrhages and intense perivascular round-cell infiltration; sections from the oblongata and spinal cord at various levels, from the walls of the third ventricle, and the optic chiasm showed much less marked infiltration without hemorrhages; sections of the cerebellar and frontal cortex showed infiltration of the pia.

History.-A girl, aged 17, was admitted to the Philadelphia General Hospital on March 5, 1919, and died three days later. The patient was in the service of Dr. B. F. Stahl, to whom we are indebted for the privilege of reporting this case. She was unmarried, one of a large family, all of whom are healthy. There was no history of influenza during the recent prevalent epidemic. One week before admission into the hospital she complained of double vision while working and was told by her companions that her eyes were crossed. During this week the patient suffered from headaches and vomiting.

Examination.-When first seen the patient was in a semi-conscious condition. She could be aroused sufficiently to obey simple commands, but if not continually prodded would soon lapse into a state of semi-consciousness. The upper eyelids were never elevated, giving the patient an appearance of double ptosis. A catatonic state was present, the arms and hands after placing being maintained in grotesque attitudes for some minutes. There was a moderate, but definite, rigidity of the neck. Brudzinski's sign was not present and Kernig's sign was doubtfully positive.

Horizontal nystagmus was present and was fairly well marked. The pupils were widely dilated and reacted very little to light. It was impossible to make the patient converge. She had been attending an eye clinic and a mydriatic had been used. The left external rectus was paretic. The eyegrounds were healthy. As far as could be determined no other cranial nerves were affected.

After much insistence the patient could perform the finger-to-nose test in a lackadaisical manner, but without any ataxia or tremor. The extremities were not paralyzed, although all muscular movements were slow and studied. The deep reflexes could not be obtained in the upper extremities and in the lower extremities were very weak, almost to the point of abolition. Plantar stimulation gave plantar flexion of the toes.

The temperature, by axilla, ranged from 99.3 to $101 \mathrm{~F}$., and a few hours before death it arose to 102.4 . The pulse on admission was 90 to the minute, soon rising to 140 where it remained. The respirations were from 25 to 40 to the minute. There were one or two profuse sweats and at times a goodly amount of tears without other evidence of emotionalism.

The lungs and heart presented no abnormal condition. The urine was negative, as was also the serological reaction. Lumbar puncture was per- 
formed without apparent discomfort to the patient, but unfortunately a bloody fluid was obtained, making a cell count impossible. The fluid did not contain any organisms.

Necropsy and Histologic Examination.-A necropsy was performed three hours after death. The heart muscle was flabby, but otherwise the abdominal and thoracic viscera showed nothing abnormal.

The brain and cord before section showed nothing on gross examination. They were placed in a solution of 10 per cent. formalin for ten days. Sections for histologic study were made under the supervision of Dr. W. G. Spiller in the Laboratory of Neuropathology of the University of Pennsylvania. These were from the midbrain, the pons, the oblongata, various levels of the cord, the walls of the third ventricle, the second, third, fourth and sixth cranial nerves, and from the frontal and cerebellar cortex.

On sectioning the midbrain and pons, areas of softening were visible to the naked eye in the region of the aqueduct of Sylvius.

The sections were stained by the Weigert, the hematoxylin and the thionin methods. The midbrain and pons in the neighborhood of the aqueduct showed intense perivascular round-cell infiltration and minute hemorrhages. This process was most marked around the aqueduct and decreased as the neuraxis was descended and ascended. It also faded out toward the periphery in the sections through the aqueduct. The oblongata and spinal cord at various levels showed decreasing perivascular infiltration without hemorrhages, except in the lumbar cord where one or two small hemorrhages were observed. Moderate pial infiltration was seen in the lumbar region. The walls of the third ventricle and the optic nerve at the chiasm showed the same perivascular round-cell infiltration. The third, fourth and sixth nerves showed nothing. Sections through the paracentral lobule exhibited rather marked round-cell infiltration involving both the cortex and the pia. The Betz cells were poorly stained, the nuclei were eccentric and the substance of the cell bodies was granular. In the frontal and cerebellar sections nothing was visible, except pial infiltration, the cortex appearing normal.

This case was clearly one of polioencephalitis similar to cases of epidemic encephalitis which have been described by Economo, ${ }^{2}$ Netter, ${ }^{3}$ Kinnier Wilson, ${ }^{4}$ Bassoe" and others.

The cases here recorded were probably of the same pathologic character, only differing in the location, severity and destructiveness of the lesions present. After all, the symptomatology of the fatal and nonfatal cases differs chiefly in the evidences of the extension and restriction of the lesions probably present. The existence of lethargy

2. Economo: Verein f. Psych. u. Neur. in Wien, April 17, 1917; Wien. klin. Wchnschr., May 10, 1917.

3. Netter: Soc. méd. des Hôpitaux de Paris, March 22, April 12-19-26, May 3, 1918; Bull. de l'Acad. de méd., May 7, 1918.

4. Wilson, Kinnier: Brain, Lond., 1906; Lancet, July 6, 1918.

5. Bassoe: J. A. M. A. 72: (April 5) 1919. 
or stupor or semi-stupor in our sixth case, as in other cases, may have been conditioned, as has been suggested by several observers, by the intensity of the pathologic process in the region of the midbrain. In other words, the cases of encephalitis with lethargy have largely the symptomatology of polioencephalits superior. Whether the etiologic factor in cases such as those here recorded is the same as that in epidemic poliomyelitis still remains to be determined. 